\title{
The Cherenkov Telescope Array
}

\section{Science Goals and Current Status}

\author{
The CTA Consortium ${ }^{1, a}$, represented by Rene A. Ong ${ }^{2, b}$ \\ ${ }^{1}$ See https://www.cta-observatory.org/consortium_authors/authors_2018_09.html \\ ${ }^{2}$ Department of Physics and Astronomy, University of California, Los Angeles CA, 90095, USA
}

\begin{abstract}
The Cherenkov Telescope Array (CTA) is the major ground-based gamma-ray observatory planned for the next decade and beyond. Consisting of two large atmospheric Cherenkov telescope arrays (one in the southern hemisphere and one in the northern hemisphere), CTA will have superior angular resolution, a much wider energy range, and approximately an order of magnitude improvement in sensitivity, as compared to existing instruments. The CTA science programme will be rich and diverse, covering cosmic particle acceleration, the astrophysics of extreme environments, and physics frontiers beyond the Standard Model. This paper outlines the science goals for CTA and covers the current status of the project.
\end{abstract}

\section{Introduction}

The field of very high-energy gamma-ray (VHE, E > $20 \mathrm{GeV}$ ) astronomy has come of age in recent years, due to a wealth of information from both ground-based and space-borne telescopes. Prior to 2000, the general expectation for the field was to detect the accelerators of the high-energy cosmic rays, most likely supernova remnants (SNRs). However, the reality over the last two decades has been the discovery of an astonishing variety of VHE gamma-ray sources, both within the Milky Way, e.g. SNRs, pulsars, pulsar wind nebulae (PWNe), cosmic ray bombarded molecular clouds, stellar binaries, massive stellar clusters, and the supermassive black hole Sgr A*, and outside, e.g. BL Lac objects, flat-spectrum radio quasars, radio galaxies, starburst galaxies, Milky Way satellites, and gamma-ray bursts (GRBs), see [1]. Most of these discoveries have been made by the major groundbased imaging atmospheric Cherenkov telescopes (IACTs), H.E.S.S., MAGIC, and VERITAS, that have been joined in recent years by the air shower array HAWC. Important contributions have also come from the space instruments Fermi-LAT and AGILE.

The recent results have been exciting and have established the field, but there are indications that they represent the tip of the iceberg and that more source classes and many more sources remain to be discovered. In addition, we know that the potential of the imaging atmopsheric Cherenkov technique has not yet been fully exploited and that significant improvements in sensitivity and resolution over existing instruments can be achieved with an array of Cherenkov telescopes that is both larger (to

\footnotetext{
ae-mail: info@cta-observatory.org

be-mail: rene@astro.ucla.edu
} 
provide more views of the shower) and more capable (e.g. with wider field-of-view and higher resolution cameras). Thus, there is both strong scientific and technical motivation for a next-generation observatory that further develops the imaging atmospheric Cherenkov technique.
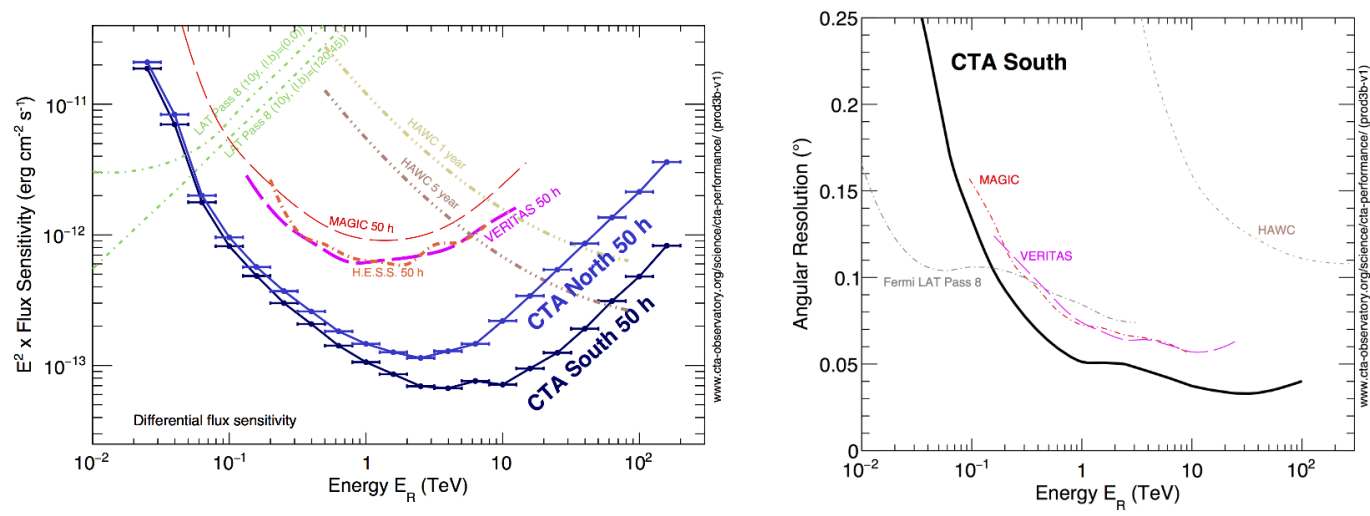

Figure 1. Left: Differential gamma-ray flux sensitivity (multiplied by $E^{2}$ ) for CTA-South and CTA-North as a function of energy. Also shown are curves for the existing IACTs (H.E.S.S., MAGIC and VERITAS), the air-shower experiment HAWC, and the Fermi-LAT space telescope. Right: Gamma-ray angular resolution for CTA-South as a function of energy, compared to existing instruments. For details on the sensitivity and angular resolution calculations and comparisons, see [2].

The Cherenkov Telescope Array (CTA) will transform our understanding of the VHE universe and will address important questions in fundamental physics, such as the nature of dark matter. It will also be an important member of the suite of experiments and observatories participating in the expanding areas of multi-wavelength and multi-messenger astronomy. Compared to the existing IACTs, CTA will: 1) cover a wider energy range, 2) have a significantly larger field-of-view, and 3) achieve up to an order of magnitude improvement in sensitivity (see Figure 1). Angular resolution and energy resolution will also be improved and full-sky coverage will be ensured by arrays in both the southern and northern hemispheres. CTA will also be the first open observatory to operate in the VHE waveband, with approximately $50 \%$ of the observing time set aside for guest observer proposals and all high-level data available to the public after a proprietary period (typically one year).

CTA has been in development for more than a decade. The concept was originated by the CTA Consortium, currently consisting of around 1,400 scientists from 31 countries. The Consortium has also developed the core science programme and has led the prototyping of telescope hardware. The CTA Observatory (CTAO) has also been established; it is the legal entity that will oversee the construction and operation of the observatory. Given a project the size and scope of CTA, international partnerships and good cooperation between the different stakeholders involved in the project (e.g. funding agencies, national laboratories, and scientific communities) are required.

\section{CTA Key Science}

The core programme of science for CTA consists of major legacy studies, called Key Science Projects (KSPs), to be carried out by the Consortium using guaranteed time (comprising approximately $40 \%$ of the observing time during the first ten-year period). The KSPs have been developed and refined by Consortium members, with extensive input from scientists from outside of CTA. The programme will 
be refined further as the observatory moves closer to its operational phase. The currently proposed KSPs are described in an extensive document that has been published as a book [3].

The core programme comprises three main scientific themes: 1) Cosmic Particle Acceleration: How and where are particles accelerated, how do they propagate and what is their impact on the environment? 2) Probing Extreme Environments: Understanding the physical processes close to neutron stars and black holes, characterizing relativistic jets, winds and explosions, and exploring radiation fields and magnetic fields in cosmic voids. 3) Physics Frontiers: What is the nature of dark matter and how is it distributed? Do axion-like particles exist? Is the speed of light a constant for high-energy photons? These themes are developed fully within nine KSPs and the Dark Matter Programme. Given space limitations, only highlights of a few KSPs are discussed here; see [3] for more complete information.

\subsection{Dark Matter Programme}

The existence of dark matter is well established from a variety of astrophysical and cosmological measurements. CTA will search for dark matter via the indirect detection technique, looking for gammaray signatures from the annihilation or decay of weakly interacting massive particles (WIMPs). The astrophysical targets for CTA are the Galactic centre halo, dwarf spheroidal galaxies, the Large Magellanic Cloud and galaxy clusters. The programme strategy focuses on maximizing the possibility of a detection. Accordingly, the primary target will be the Galactic centre halo that houses the largest concentration of dark matter in our Galaxy, with secondary data taken on the other targets. With a deep $500 \mathrm{~h}$ observation of the Galactic centre halo, CTA will achieve a sensitivity level below the benchmark relic cross-section over a wide WIMP mass range that extends into the multi-TeV region (see Figure 2). Thus, CTA will be an important player in the world-wide search for dark matter, providing unique and complementary information to direct-detection experiments and the Large Hadron Collider.
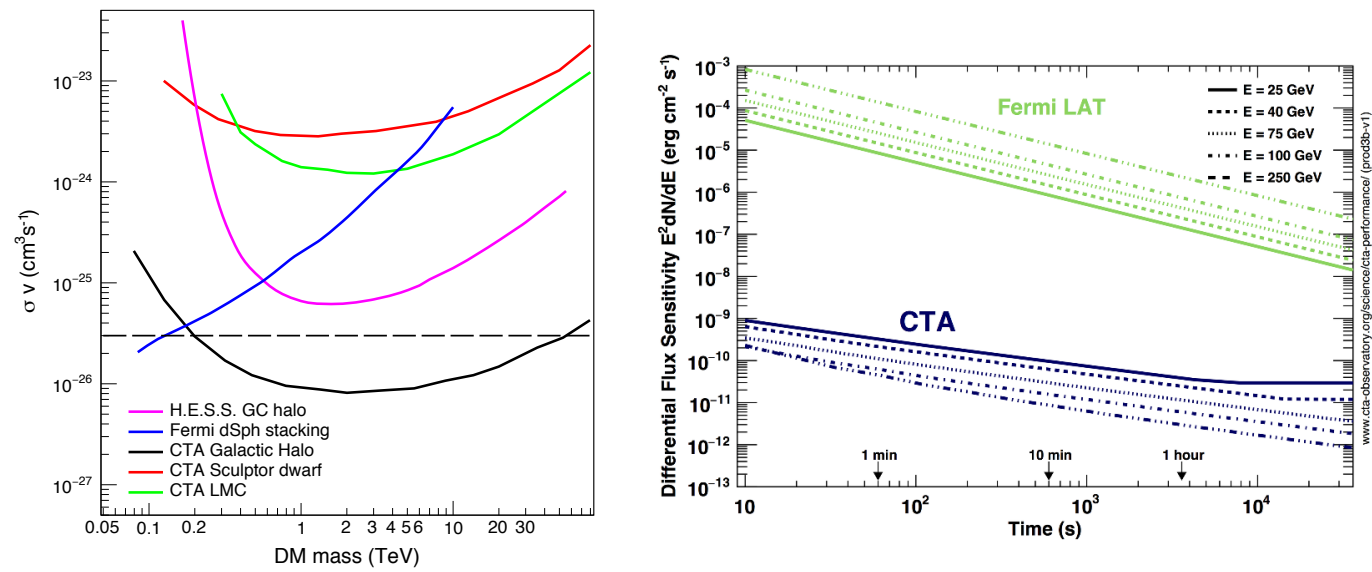

Figure 2. Left: Predicted CTA sensitivity on the velocity-weighted cross section $(\langle\sigma v\rangle)$ for dark matter annihilation for various targets, as indicated in the legend. Results from H.E.S.S. on the Galactic centre halo [4] and from Fermi-LAT on dwarf spheroidal galaxies [5] are also shown; see [3] for details. Right: Flux sensitivities for CTA and Fermi-LAT at selected energies as a function of observing time; see [2] for details. 


\subsection{Transients KSP}

CTA is a powerful instrument for exploring the transient universe. With its very large collection area and high signal to noise, CTA has outstanding instantaneous sensitivity over a broad energy range, giving it a distinct advantage over Fermi-LAT at lower energies and HAWC at higher energies. For transient time scales of a day or less, CTA is at least several orders of magnitude more sensitive than Fermi-LAT (see Figure 2). CTA's disadvantage is that it has a limited field-of-view compared to Fermi-LAT or HAWC; hence, reaction time for CTA is critical and its transients programme is focused on follow-up observations. The Transients KSP is an integrated programme that encompasses a variety of multi-wavelength and multi-messenger alerts, including explosive transients such as GRBs and gravitational wave events, active galactic nuclei (AGN) flares, Galactic transients, and high-energy neutrino alerts. Rapid dissemination to the wider community of the VHE properties of transients observed by CTA is an important component of the KSP.

\subsection{Galactic Plane Survey KSP and Cosmic Ray PeVatrons KSP}

Surveys of the Galactic plane are key legacy programmes for all major observatories. Previous surveys in the VHE gamma-ray band have been carried out by both ground-based and space-borne instruments. The CTA Galactic Plane Survey (GPS) will be the first high sensitivity, high angular resolution survey carried out at TeV energies. Using $1600 \mathrm{~h}$ of observing time, the GPS KSP will cover the entire Galactic plane with a graded-exposure strategy, emphasizing key regions of the Galaxy in both the southern and northern hemispheres. From simulations and our (incomplete) knowledge of the VHE source population, we expect the GPS to discover many hundreds of new sources, especially PWNe, SNRs, and binaries. The GPS will also have great potential for the discovery of new, unexpected phenomena and will provide a detailed view of diffuse VHE gamma-ray emission. Figure 3 shows a simulated image of the GPS results.
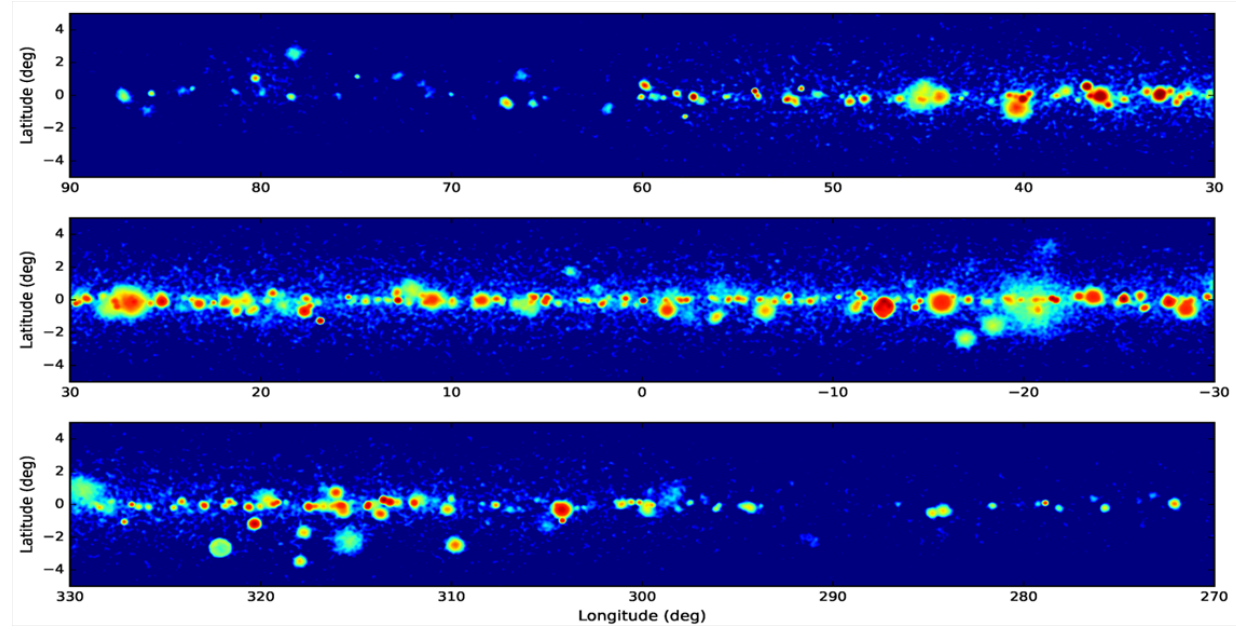

Figure 3. Simulated results from the CTA Galactic Plane Survey in very high-energy gamma rays for half of the plane. The simulation incorporates the proposed GPS observation strategy and a source model containing SNR and PWN populations, as well as diffuse emission.

A long-standing issue in high-energy astrophysics is to understand what sources accelerate cosmic ray nuclei (hadrons) to the knee in the energy spectrum. The standard paradigm involves diffusive 
shock acceleration in SNRs, but so far only a handful of SNRs provide good evidence for hadronic acceleration and their energy spectra reach only to $\sim 10 \mathrm{TeV}$. The goal of the Cosmic Ray Pevatrons $\mathrm{KSP}$ is to determine the sources capable of accelerating hadrons to PeV energies. For this, the GPS will be used as a finder and the five brightest sources with spectral extension out to $\sim 100 \mathrm{TeV}$ will be targeted for deep follow-up observations. For any PeVatrons detected, correlation with data from other wavebands will be crucial for clear identification of the physical sources.

\subsection{Extragalactic Survey KSP}

The motivation for a survey of the extragalactic sky is similar to that for the GPS; we wish to observe a large portion of the sky in an uniform manner to get an understanding of the dominant source population (in this case AGN) and to have sensitivity to completely unexpected phenomena. In the Extragalactic Survey KSP, one-quarter of the sky will be surveyed to a limiting sensitivity of $0.6 \%$ of the (standard candle) Crab nebula flux. The survey will be carried out by both CTA-South and CTANorth and will connect to the GPS and cover the important regions of Coma, Virgo, Cen A, and the northern Fermi Bubble. The key science targeted by the survey includes the unbiased determination of the luminosity function for AGN, the discovery of VHE emission from as yet undetected source classes such as Seyfert galaxies and ultraluminous infrared galaxies, and the detection of GRBs in the prompt phase. Studies are ongoing to understand the potential of carrying out the survey using a divergent pointing strategy (in which various telescopes are pointed to sky positions slightly offset from the target position), which would allow for larger instantaneous field-of-view.

\section{CTA Design and Current Status}

\subsection{CTA Design}

CTA will consist of two large arrays of imaging atmospheric Cherenkov telescopes. The larger southern array, CTA-South, will be constructed in the Atacama Desert of Chile at an elevation of 2,100 m, close to the existing Paranal Observatory of the European Southern Observatory. CTA-North, will be built at an elevation of 2,200 m on the island of La Palma, Spain, located within the existing Observatorio del Roque de los Muchachos of the Instituto de Astrofisica de Canarias. Two important administrative/scientific centers will be located in Europe: the CTA Headquarters in Bologna, Italy and the CTA Science Data Management Center in Zeuthen, Germany.

In order to optimize the scientific capability within a constrained funding envelope, CTA will employ three different sizes of telescopes [6]. Four Large-Sized Telescopes (LSTs), with mirror diameters of $23 \mathrm{~m}$, will provide the sensitivity in the lowest energies of the CTA waveband (down to $20 \mathrm{GeV})$, where the Cherenkov photon density in gamma-ray air showers is low $\left(\sim 1 / \mathrm{m}^{2}\right)$. MediumSized Telescopes (MSTs), with mirror diameters of 9-12 m, will be distributed around the LSTs to provide sensitivity in the core energy range of $100 \mathrm{GeV}-10 \mathrm{TeV}$. Small-Sized Telescopes (SSTs), with mirror diameters of $\sim 4 \mathrm{~m}$, will extend the size of CTA-South and provide sensitivity to energies of $300 \mathrm{TeV}$ and above. SSTs are only envisioned for the southern array for which the entire inner regions of the Galactic plane will be visible. For both the MSTs and the SSTs, prototypes are being developed that use either the conventional single-mirror Davies-Cotton optical design or the newly developed dual-mirror Schwarzschild-Couder optical design. In the baseline CTA design, CTA-South will comprise 4 LSTs, 25 MSTs, and 70 SSTs, covering an area of $\sim 5 \mathrm{~km}^{2}$, and CTA-North will comprise 4 LSTs and 15 MSTs, covering an area of $\sim 0.5 \mathrm{~km}^{2}$. The proposed telescope array layouts, shown in Figure 4, were derived through a lengthy and comprehensive simulation process [7]. 

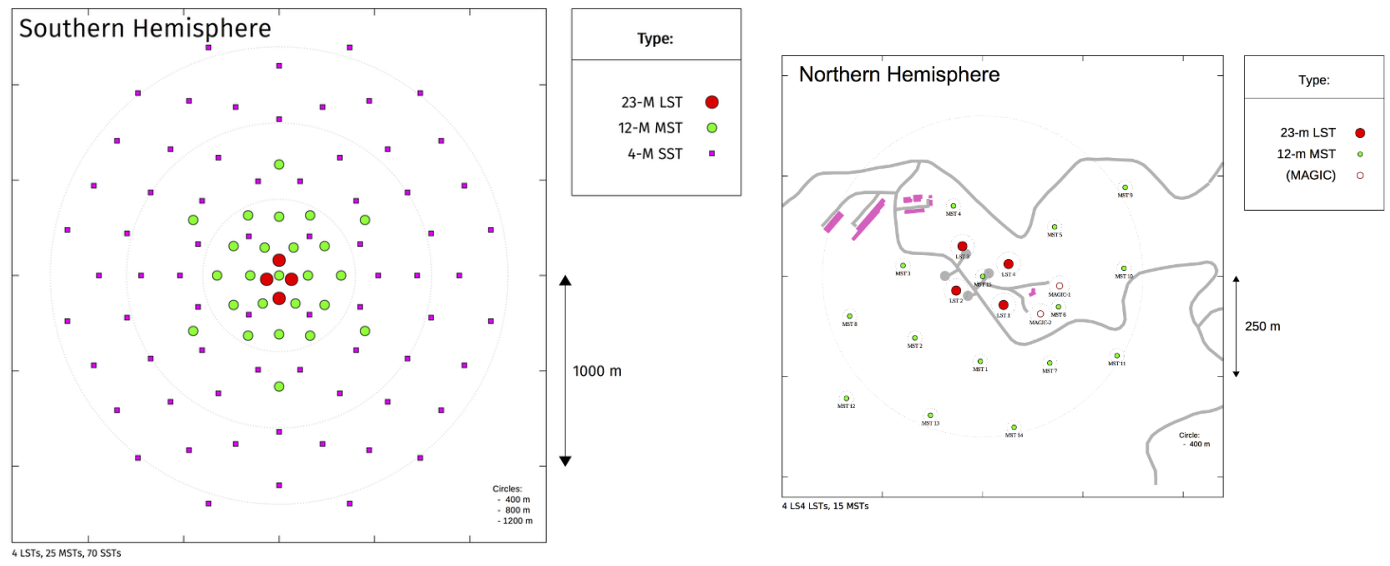

Figure 4. Left: Proposed layout for the three sizes of telescopes (LSTs, MSTs, and SSTs) in CTA-South (Paranal, Chile). Right: Proposed layout for the two sizes of telescopes (LSTs and MSTs) in CTA-North (La Palma, Spain). The roads are shown in grey and the buildings in purple. The current MAGIC telescopes are indicated by the open red circles.

\subsection{CTA Status}

Initial planning for CTA started as early as 2005, when the community came together at Palaiseau, France to discuss the future of ground-based gamma-ray astronomy [8]. Since then, an enormous amount of development work has been carried out and CTA has been heavily reviewed and endorsed by the major roadmap committees in particle physics, astroparticle physics, and astronomy (see, e.g., [9-11]). Most recently, CTA was promoted to Landmark status in the European Strategy Forum on Research Infrastructure (ESFRI) 2018 Roadmap [12]. The management of CTAO has been established. The CTA Council takes care of overall governance and the Project Office manages the construction project. An interim legal entity (CTAO gGmbH), composed of shareholders from eleven countries and associate members from two countries, has been established for the preparation of the implementation of CTA.

The development of telescope hardware is being carried out by the Consortium institutes, as inkind contributions to the observatory. Substantial software development has also started, both at the Project Office and within Consortium institutes. As shown in Figure 5, six full prototype telescopes exist and are being actively tested. Work is now focused on design optimization, with the goal to reduce the number of telescope and camera options. Site hosting agreements for both CTA sites have been signed and site preparatory work is ongoing. In this context, CTA-North is more advanced since much of the necessary infrastructure already exists. The first telescope to be located at a CTA site is the prototype LST that was inaugurated at the CTA-North site in October 2018 and is now undergoing commissioning. Current plans are: start of construction in 2019, first pre-production telescopes on site in 2020 and completion of construction in 2025. Since the CTA design is quite modular, initial operations can start as early as 2023 , using a portion of the array available at either site.

\section{References}

[1] TeVCAT: http://tevcat.uchicago.edu/ 


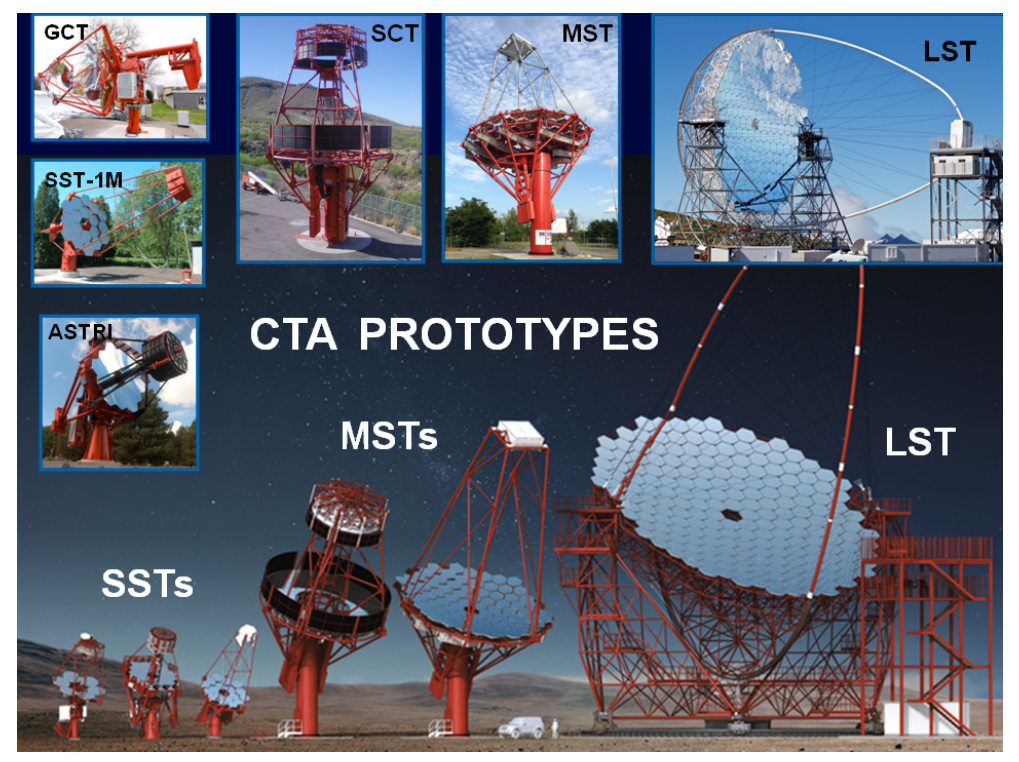

Figure 5. Concept drawings and photographs of CTA protoype telescopes, as of 2018. Clockwise (from lower left): SST-ASTRI at Mt. Etna, Italy, SST-1M at Krakow, Poland, SST-GCT at Meudon, France, SCT at Whipple Observatory, AZ, USA, MST at Adlershof, Germany and LST-1 at La Palma, Spain.

[2] https://www.cta-observatory.org/science/cta-performance/

[3] The CTA Consortium, Science with the Cherenkov Telescope Array (World Scientific Publishing, 2019), ISBN 978-981-3270-08-4, arXiv: 1709.07997, DOI: 10.1142/10986

[4] H. Abdallah, A. Abramowski, F. Aharonian, F. Ait Benkhali, A.G. Akhperjanian, E. Angüner, M. Arrieta, P. Aubert, M. Backes, A. Balzer et al., Physical Review Letters 117, 111301 (2016), 1607.08142

[5] M. Ackermann, A. Albert, B. Anderson, W.B. Atwood, L. Baldini, G. Barbiellini, D. Bastieri, K. Bechtol, R. Bellazzini, E. Bissaldi et al., Physical Review Letters 115, 231301 (2015), 1503.02641

[6] B.S. Acharya, M. Actis, T. Aghajani, G. Agnetta, J. Aguilar, F. Aharonian, M. Ajello, A. Akhperjanian, M. Alcubierre, J. Aleksić et al., Astroparticle Physics 43, 3 (2013)

[7] The CTA Consortium, Astroparticle Physics (2019), submitted

[8] B. Degrange, G. Fontaine, eds., Proceedings, 7th Workshop on Towards a Network of Atmospheric Cherenkov Detectors 2005, Ecole Polytechnique (Ecole Polytechnique, Palaiseau, France, 2005)

[9] The AStroParticle ERAnet (ASPERA) roadmap: http://www.aspera-eu.org/

[10] The U.S. Astronomy and Astrophysics Decadal Survey (ASTRO2010): http://www. nationalacademies.org/astro2010

[11] The U.S. HEPAP P5 Panel: http://usaparticlephysics.org/p5/

[12] The ESFRI 2018 Roadmap: http://roadmap2018.esfri.eu/ 